\title{
Giuliano Boccali, Raffaele Torella, (éds.), Passioni d'Oriente. Eros ed emozioni in India Tibet
}

Torino, Giulio Einaudi editore, coll. «Piccola Biblioteca Einaudi», 2007, 244p.

\section{André Padoux}

\section{(2) OpenEdition} Journals

Édition électronique

URL : http://journals.openedition.org/assr/21519

DOI : $10.4000 /$ assr.21519

ISSN : $1777-5825$

Éditeur

Éditions de l'EHESS

Édition imprimée

Date de publication : 31 décembre 2009

Pagination : $75-342$

ISBN : 978-2-7132-2218-4

ISSN : 0335-5985

\section{Référence électronique}

André Padoux, «Giuliano Boccali, Raffaele Torella, (éds.), Passioni d'Oriente. Eros ed emozioni in India Tibet », Archives de sciences sociales des religions [En ligne], 148 | octobre-décembre 2009, document 148-23, mis en ligne le 27 janvier 2010, consulté le 21 septembre 2020. URL : http:// journals.openedition.org/assr/21519; DOI : https://doi.org/10.4000/assr.21519

Ce document a été généré automatiquement le 21 septembre 2020.

(C) Archives de sciences sociales des religions 


\section{Giuliano Boccali, Raffaele Torella, (éds.), Passioni d'Oriente. Eros ed emozioni in India Tibet}

Torino, Giulio Einaudi editore, coll. «Piccola Biblioteca Einaudi», 2007, 244p.

\section{André Padoux}

\section{RÉFÉRENCE}

Giuliano Boccali, Raffaele torella, (éds.), Passioni d'Oriente. Eros ed emozioni in India

e Tibet, Torino, Giulio Einaudi editore, coll. «Piccola Biblioteca Einaudi», 2007, 244p.

1 La pensée philosophique indienne, toujours restée ancilla theologiae, car inséparable de systèmes socioreligieux, a la réputation hors de l'Inde (mais d'ailleurs aussi sur place) d'être toute orientée vers la recherche du salut par le renoncement au monde et l'ascétisme. Mais l'Inde, foyer toujours actif depuis quelque trois mille ans de tant de formes religieuses et de subtiles spéculations métaphysiques, est aussi une terre où des divinités se distinguent par leurs performances sexuelles, où un phallus dressé représente un des principaux dieux; c'est le pays du Kāmasūtra et des poèmes érotiques, celui, enfin, de l'exaltation de l'effervescence cosmique, de la représentation de la beauté des corps nus, des recherches les plus poussées sur la jouissance esthétique - celui, en somme, où tout ce qui est passion (rāga), quête de la saveur (rasa), ne cesse d'être présent. En fait, c'est peut-être surtout dans cet aspect - dont traite cet ouvrage - que se trouve ce qu'il y a de plus essentiellement indien, et c'est là ce que veut faire ressortir ce recueil d'études dues à d'excellents spécialistes, ce qui en fait un ouvrage extrêmement intéressant et, pour nous Occidentaux, très révélateur.

La première partie de l'introduction, «Entre renonciation et amour ", envisage la tension entre ces deux tendances telle qu'elle apparaît dans divers aspects de la culture indienne: quelle place tiennent les passions dans l'évolution spirituelle de l'être 
humain dans la perspective de sa fin ultime - la libération des liens de l'existence (moks a/mukti ou nirvāna a) - alors qu'elles sont considérées a priori, et quasiment partout désignées, comme le principal obstacle à cette fin à laquelle chacun doit (s'il en est capable) aspirer? Or, si le principe de la nécessité de renoncer aux plaisirs du monde afin de parvenir au salut est généralement posé, on s'aperçoit, si l'on étudie les textes, que cette position extrême n'est pas sans être contredite par divers aspects du comportement traditionnellement prescrit dans le monde hindou. Ainsi, la règle généralement valable des étapes de la vie d'un hindou de caste (les āśrama) ne prescrit de formes d'ascèse ou de renoncement qu'au début et à la fin de l'existence, le reste étant jouissance du monde, cela alors que le but ultime du salut y reste toujours valable. La tradition hindoue, de même, ne fixe que trois buts de vie ou de forme d'activité à l'être humain : kāma d'abord, la jouissance ou poursuite des désirs ou passions, artha, la recherche de la richesse et dharma, la défense ou pratique des règles régissant la société. S'y ajoute certes un quatrième but, mokșa, la libération, mais, si celui-ci est le plus élevé, il n'est pas formellement prescrit - et pas nécessairement très désiré. La vie en ce monde et ses plaisirs, avec l'espoir d'une renaissance favorable, est donc la règle. (C'était d'ailleurs la vision de l'Indien védique - même Manou (5.56) ne voyait pas de mal à manger de la viande, boire des spiritueux et à se livrer à l'amour «dans les cas où cela est permis »). Des exemples littéraires nombreux (certains cités ici) montrent le poids en réalité essentiel de la vie en ce monde et de la jouissance de ses satisfactions - parmi lesquelles celles de la vie amoureuse et des plaisirs de la chair. Typique à cet égard est le Kāmasūtra, objet du premier des articles ici rassemblés, dont il se trouve que l'auteur, Vatsyayāna, a été un renonçant : on voit là, dans la tradition hindoue, se rejoindre le rôle de l'ascète renonçant et la place faite à ce qui relève du kāma, le désir. Ce dernier va, avec le tantrisme, être posé comme une des voies possibles vers la libération - mais une libération qui n'est plus renoncement mais plénitude, faisant appel à tout ce qu'offre la création divine pour parvenir à sa source, dépassant le monde créé, mais en en utilisant les moyens, les bonheurs, pour le transcender. L'appel n'étant d'ailleurs pas seulement aux joies sensuelles, mais aussi, et surtout, dans le domaine tantrique, aux forces obscures, à la puissance de la transgression, lors de cultes extatiques de divinités d'autant plus puissantes (et donc efficaces pour aller vers le salut) qu'elles incarnent des forces dangereuses, mais qu'il est possible pour l'adepte initié de se rendre favorables et même de manipuler et d'en utiliser par des rites appropriés les possibilités salvatrices. G. Boccali et R. Torella soulignent également la place qu'a, particulièrement dans le domaine tantrique, l'expérience esthétique, jouissance intellectuellement vécue $\mathrm{du}$ beau, dont le point le plus haut, l'émerveillement extasié (camatkāra, en sanskrit), est une expérience extatique du beau. (Elle peut l'être aussi du terrible ou de la douleur.) Il s'agit toujours, notent-ils (en citant Marcel Proust), d'une transcendance du temps.

Dans la deuxième partie, "Passioni ed emotioni nelle filosofie e nelle relogioni dell'India », G. Boccali et R. Torella font d'abord remarquer que, curieusement, «alors que l'Inde s'est toujours montrée avide d'analyser tous les champs du connaissable, elle n'a jamais conçu comme une discipline autonome un champ d'étude tel que notre psychologie ». Ce n'est, de fait, que dans les textes de cosmologie, de métaphysique ou de yoga, d'esthétique (ou même de grammaire) que nous trouvons de souvent fines, ingénieuses, observations d'ordre psychologique. Dans les textes sanskrits traitant de la situation de l'homme en ce monde, dans la littérature philosophico-religieuse élaborée par les brahmanes (à leur usage), on voit généralement posées comme à l'origine de 
l'univers autant que dans son fonctionnement (et donc dans la situation de l'être humain) une dichotomie essentielle : d'une part, le monde de la nature (prak區ti), dont relève tout ce qui fait l'être humain - corps, sens, émotions, facultés intellectuelles -, monde qui se perpétue par le jeu de ses propres forces, et, d'autre part, ce qui relève de l'esprit, qui est divin et qui, autonome, immobile, brille dans une pure solitude. Cette vision dichotomique est, sous différentes formes et en dévaluant plus ou moins le corps, celle des systèmes (darśana) traditionnels hindous; celle également, à leur manière, du bouddhisme et du jainisme. C'est la prévalence de ces conceptions qui a donné la vision que l'Occident a longtemps eue de l'Inde comme «d'un monde ascétique, désincarné, en même temps que prisonnier d'une infinie prolifération de couleurs, odeurs et sons - de la vie dans toutes ses formes les plus splendides et fugaces $»$ (p. 28).

Mais l'Inde, disent G. Boccali et R. Torella, n'est pas cela. Elle ne l'était pas dans les débuts, aux temps védiques. Elle ne le sera pas plus tard avec les développements de la dévotion (la bhakti) dont tous les aspects ne sont pas ascétiques. Elle ne l'a jamais été dans la vie courante des Indiens qui (sauf chez les brahmanes, infime minorité de la population) est liée aux anciens cultes populaires, autochtones. Elle n'est pas, enfin, dans ce qui a formé ce qu'il y a de plus vivant, intéressant et créateur, à partir des premiers siècles de notre ère, dans la pensée et la pratique religieuse indiennes: le tantrisme, dont cet ouvrage va montrer quelques aspects. G. Boccali et R. Torella évoquent alors brièvement ce qu'est l'approche tantrique du monde et de l'être humain, citant d'abord un surprenant verset d'un traité à la gloire de la Déesse, du grand philosophe tantrique Abhinavagupta (c. 975-1025) où il est dit: «L'homme de désir peut tout atteindre $\mathrm{du}$ moment où tout a pour base ou principe ultime précisément le désir. » Cette "énergie du désir », disent nos deux auteurs d'une façon toute tantrique, « est celle qui, pour le śivaïsme tantrique (le tantrisme est avant tout śivaïte), se manifeste dans la jouissance et la joie des sens qui donne vie au bouillonnement des émotions, lequel atteint son point le plus haut dans la passion amoureuse. De cette énergie, tout l'univers est pénétré. Elle est l'unique matrice de tout dynamisme et de toute vie, traversant de son courant aussi bien les plus subtiles abstractions de la pensée que le modeste train-train de la vie quotidienne. Le premier culte que le fidèle [tantrique] doit rendre l'est aux déesses de sa propre conscience (svasamvid-devī) qui ne sont rien d'autre que les divinités des sens (karaṇeśvarī), les Maîtresses de ses sens ; l'offrande sacrificielle se compose dès lors de tout ce qui fait la vie ordinaire - qui, ainsi, est tout autre qu'"ordinaire"." Selon un tantra śivaïte important, le Mālinivijayottaratantra (15.44) : «les sens sont la cause des liens aussi bien que de la libération, disent les sages : liés, ils lient, libérés, ils donnent la libération. » C'est ainsi toute une vie différente, libre, fondue avec l'énergie divine, qui s'ouvre à l'adepte. Mais on ne peut guère rendre compte ici de la richesse (toute tantrique !) de cette introduction.

5 Les sept études qui la suivent en reprennent certains thèmes à l'occasion de cas particuliers. Là encore, on ne peut guère rendre justice à la richesse de certaines d'entre elles. La première, de Cinzia Pieruccini (pp.33-60), est consacrée à ce texte classique de l'érotisme indien qu'est le Kāmasūtra, généralement imaginé chez nous comme n'étant qu'un manuel de pratiques sexuelles, alors qu'il est tout autre chose. Le kāma, comme on l'a vu, s'il est l'eros, est aussi et surtout (avec artha et dharma) une des trois fins de la vie humaine: il concerne tout ce qui est jouissance des plaisirs de ce monde, participation à ses joies. S'il fait effectivement une place aux techniques 
érotiques, le Kāmasūtra est surtout un manuel d'art de vivre à la recherche du plaisir pour un citadin oisif et fortuné dont la vie est conditionnée par les règles de la société des castes sans ignorer toutefois la variété possible des comportements et des plaisirs (qui ne sont pas tous nécessairement classiquement hétérosexuels). La conception de la vie y est évidemment toute masculine: la femme y est un moyen, même s'il importe de tenir compte de sa sensibilité et si son plaisir n'y est pas ignoré. Elle est, dans ce traité, l'interlocutrice la plus parfaite de l'homme de goût: la courtisane y est un modèle d'élégance, de culture, de raffinement. Elle apparaît, en fait, comme la figure féminine la plus autonome (elle est la «jamais veuve »). Et il est à noter qu'elle n'a pas cessé de l'être à certains égards jusqu'aux temps modernes.

6 L'article qui suit, de R. Torella, « Il tantrismo hindu e l'invenzione del desiderio ", est à mon sens, avec l'introduction, la contribution la plus intéressante et ingénieuse à ce livre. L'argumentation (où s'exprime un indianiste éminent) ne peut pas se résumer utilement. Disons seulement que R. Torella note la place importante faite au désir (kāma) dans le Mahābhārata et dans certaines Upaniśads et même dans le Rgveda, donc dès une époque ancienne. Par la suite, diverses conceptions tantriques, dont celle des « cuirasses » qui limitent l'être humain (les kañcuka) dont celui de rāga, l'attachement passionnel, montrent comment l'être désirant qu'est l'homme s'insère dans le cosmos et a part à l'effervescence énergétique qui le fait apparaitre et l'anime. Il y a dans cette étude plusieurs des observations les plus pertinentes qui aient été faites sur la nature et les caractéristiques du phénomène tantrique hindou.

7 Vient ensuite une étude de Malgorzata Sacha portant sur la déesse Kālī (associée au dieu Śiva), déité particulièrement présente sous diverses formes dans l'univers tantrique, des époques anciennes à nos jours. Redoutable, destructrice, associée à la mort, elle est aussi perçue par ses dévots comme protectrice, favorable, prenant donc les formes contradictoires (mais parfois associées) de la pulsion passionnelle. C'est de la place des passions dans le théâtre et la littérature sanskrite que traite G. Boccali. Il le fait attentivement, mais ce sont là des aspects bien connus en Occident où une des premières œuvres littéraires indiennes connue fut le drame amoureux de Sakuntala. L'article de David Smith aborde "la représentation des émotions dans la sculpture indienne. Le corps, l'esprit»: soumises à des canons iconographiques stricts, ces œuvres semblent en réalité n'être guère transmetteuses d'émotions, mais elles peuvent en être le support, l'occasion, quand elles représentent, « incarnent », des divinités. Les exemples donnés (parfois illustrés) sont intéressants.

8 Passioni d'Oriente ne pouvait guère laisser de côté l'autre grande création religieuse de l'Inde, le bouddhisme qui, en outre, s'est étendu en Orient bien au-delà du souscontinent indien - cela même s'il prêche avant tout, pour arriver au salut, l'extinction des passions. Giacomella Orofino présente ainsi des «Sentiers de la libération dans le bouddhisme indo-tibétain", où certaines pratiques ascétiques incluent parfois une union sexuelle rituelle. Mais celle-ci semble davantage utiliser la force de la pulsion sexuelle mise en jeu afin de la dépasser vers l'arrêt et la transcendance que pour la vivre comme passion. Les cas mentionnés relèvent de la tradition du Dzog-Chen, la Grande Perfection, qui aurait été proche de certains mouvements tantriques Śivaïtes. On sait, de fait, qu'une part appréciable du canon bouddhique tibétain est constitué de traductions (de «mis-translations", dit le professeur Sanderson!) de textes śivaïtes : dans le domaine tantrique, on est toujours, pour parler comme Sanderson, dans le "Śaiva age " - dans le monde śivaïte. Les directeurs de l'ouvrage ont tenu enfin à 
inclure dans leur tableau des passions d'Orient, le bouddhisme theravāda, avec, pour finir, une étude de Corrado Pensa, «Conscience, émotions, équanimité ». Mais, malgré toute la science de cet excellent spécialiste, les émotions ne paraissent guère jouer un rôle important dans la méditation bouddhique. (Si l'on était méchant, on dirait qu'elles apparaissent surtout de nos jours dans le comportement tout sauf non violent des bonzes de Sri Lanka...)

9 Cet excellent petit volume sait, à l'italienne, s'adresser de façon accessible au grand public sans sacrifier la précision parfois érudite. C'est une grande qualité. Il contribue en outre à explorer le domaine tantrique, champ de l'importance et de l'intérêt duquel on n'a, malgré quelques pionniers, que récemment pris conscience et dont l'exploration est encore en cours. Mais ces études sont, sauf exception (cf. Torella, Il pensiero dell'India, dans ce même numéro : Arch. 148-127), inaccessibles au grand public cultivé, laissant la place aux publications les plus fantaisistes et douteuses. Une étude d'ensemble accessible, en français, sur le sujet reste encore (en 2009) à paraître. D'où la valeur de ce recueil, qui mériterait grandement d'être rendu accessible, par une traduction, à ceux qui ne savent pas l'italien. 